\title{
Antinociceptive activity of Channa striatus (haruan) extracts in mice
}

\begin{abstract}
Haruan, Channa striatus, is a snakehead fish consumed in many parts of the southeast Asian region. It is believed to promote wound healing, as well as reduce post-operative pain. In an attempt to establish the scientific basis for the alleged pain-relieving benefits of this fish, we studied the antinociceptive effects of whole fillet and mucus extracts from haruan in the mouse using the abdominal constriction and tail flick tests. In the abdominal constriction test, the 30 min fillet extract exhibited concentration-dependent inhibition of the writhing response in the $10-50 \%$ concentration range, with $20 \%$ as the IC50 value. This activity was not dependent on the duration of extraction, with no significant differences among the extracts obtained at durations of 10, 20, 30, 60, 90 and 120 min (range between 45-54\% inhibition at $20 \%$ concentration). The mucus extract also showed concentration-dependent inhibition of the abdominal constriction response - at the highest concentration used the average inhibition was $68.9 \%$, while IC50 value was $25 \%$. Neither the fillet extract (30 min, 20\%) nor the mucus extract $(25 \%)$ had any demonstrable effect on the tail flick latency on their own, but significantly enhanced the antinociceptive activity of morphine in this assay. Similarly, low concentrations of the mucus and fillet extract enhanced the effects of morphine in the abdominal constriction test. Collectively, these results suggest a scientific basis for the folklore practice of eating haruan fish in the post-operative period for pain relief: Haruan extracts have antinociceptive activity and enhance the activity of other antinociceptive agents.
\end{abstract}

Keyword: Abdominal constriction test; Acute pain; Analgesia; Opioid analgesia; Postoperative pain; Tail flick test 